\title{
Does Generalized Anxiety Disorder (GAD) Predispose the Severity and Probability of the Neuropathic Component of Back Pain? A Cross- sectional Study
}

\author{
Aditi Mukhopadhyay ${ }^{1^{*}}$ and Subhanu Sekhar Roy Chowdhury ${ }^{2}$ \\ ${ }^{1}$ Department of Physiology, Hooghly Mohsin College, The University of Burdwan, India \\ ${ }^{2}$ Department of Physiology, Lalgarh Govt. College, Vidyasagar University, India
}

"Corresponding author: Aditi Mukhopadhyay, Department of Physiology, Hooghly Mohsin College, The University of Burdwan, India, E-mail: aditi.apsara@gmail.com

Received date: September 10, 2018; Accepted date: September 28, 2018; Published date: October 5, 2018

Copyright: (02018 Mukhopadhyay A, et al. This is an open-access article distributed under the terms of the Creative Commons Attribution License, which permits unrestricted use, distribution, and reproduction in any medium, provided the original author and source are credited.

\begin{abstract}
Introduction: Back pain and GAD are very common among housewives across the globe. Various pathophysiological factors can deteriorate the prognosis and diagnosis of back pain, among which neuropathic component is notable. The present study explored whether GAD increases the perception and severity of neuropathic component of back pain.

Methods: Forty-eight housewives were included in this study. The participants had at least 5 years' experience of household work. Cross-sectional data was collected to Subjective and objective detection of back pain was done through the VAS Scale and the Pain Detect Tool respectively. GAD scores were differentially detected with the GAD 7 tool. The clinical and medication history of the participants were noted through structured-interview sessions in presence of a pain specialist.
\end{abstract}

Results: The results showed that, about one-half of the participants exhibited a neuropathic pain. GAD was shown to significantly increase the odds of severity and perception of neuropathic pain among the participants. A majority of the subjects reported that they did not receive any medications for pain relief with analgesic therapy. The regression analysis reflected that, addition of GABA-agonists and anxiolytics resulted in non-significant perception of pain on the VAS scale.

Conclusion: These findings suggest that appropriate medication could significantly reduce the perception of back pain (on VAS scale) in GAD-affected women. The study voiced the need for personalized medicine in managing back pain across patients affected with psychopathic comorbidity.

Keywords: Back pain; Neuropathic pain; GAD; Therapeutic modalities

\section{Introduction}

India being the fastest developing world economy next to China needs sufficient women contribution to the country's gross productivity in the world market to keep pace with working women of Japan and others. Back pain can significantly reduce productivity in men and women across the globe and India is no exception. Different factors such as postural, neurological and psychopathic disorders could influence the severity and perception of back pain in at-risk individuals. For example, non-neutral postures, repetitive movements, high lifting index and other lifestyle factors results in the genesis of pain. Back pain can be classified as either acute or chronic. The reported prevalence of low back pain in the study population varied between $42 \%$ [1] to $83 \%$ [2]. However, there is inconclusive evidence regarding the prevalence of back pain as a function of psychopathic disorders in the target population. However, psychopathic component such as anxiety and depression has been found to be comorbid for back pain in a number of studies $[3,4]$. The prevalence of anxiety disorders is 60.05 million (global 264 million) in the South East Asian region (WHO, 2015) including the Indian subcontinent. The prevalence of
GAD in India in $2015-2016$ is $0.38 \%$ (males) and $0.76 \%$ (females) [5]. Hence, it is speculated that the comorbid effects of psychopathy could influence the severity and perception of back pain in respective stakeholders.

A study conducted in Brazil have shown that, the patients with pain have a higher frequency of having anxiety, which is significant $(\mathrm{P}=0.02)$ and the comorbid condition of pain with anxiety worsens the health condition and quality of life of the patients. As reported by Heer et al. in 2329 subjects revealed presence of a anxiety $(\mathrm{OR}=4.84$; $\mathrm{P}<0.001)$, or co-morbid depressive and anxiety disorder $(\mathrm{OR}=30.26$; $\mathrm{P}<0.001)$ was associated with the Chronic Pain Grade, along with symptom severity was associated with more disabling and severely limiting pain. Also, a remitted depressive or anxiety disorder showed more disabling and severely limiting pain $(\mathrm{OR}=3.53 ; \mathrm{P}<0.001)$ as compared to controls.

Among all the anxiety traits, GAD is reported as the most common anxiety trait among the chronic pain sufferers. So, the objective of the present study was to investigate the influence of GAD on the symptoms, perception, and severity of chronic back pain (upper, middle and lower) in group of middle aged women in suburban districts West Bengal, India. 
Page 2 of 6

\section{Methodology}

\section{Study design and sampling}

The study was conducted as a retrospective and cross-sectional trial. A convenience sampling was undertaken to select the study participants $(\mathrm{n}=48)$. This study was conducted from September, 2017 to July, 2018. The study sample include housewives belonging from middle class families are taken $(n=48)$ from different districts of West Bengal, India within the age of 30-60 years. All participants were included post-informed consent. The study was further supported by Institutional Ethical Committee. The inclusion criteria were set up such so that every woman has the experience of active household works atleast for 5 years. The exclusion criteria were set up such, so that, there were no subjects having Depression, other traits of Anxiety, and other Musculo-skeletal disorders (previously detected by physicians) like Radiculopathy or Disc-prolapse. Subjects were controlled at baseline from confirmed cases of major depressive disorder and other clinical comorbidities.

\section{Procedure}

Subjects were interviewed in a semi-structured questionnaire basis, to explore their demographic and clinical data. The interview questionnaires include subjective and objective assessment of back pain. The subjective analyses of pain were based on the VAS scale and the objective analyses of pain were done through pain score through the Pain Detect Tool [2]. The Generalized Anxiety Disorder or GAD was detected with the GAD-7 tool [6-11]. The clinical data that were surveyed include medication history of the patients through prescription transcripts.

\section{Data analysis}

Both descriptive and inferential statistics were used to interpret the results. Exploratory factor analyses were undertaken for sampling adequacy and adequacy of the variables considered for the study. Normality tests were conducted to explore the distribution pattern of the variables with appropriate transformation. The odds-ratio for pain in the presence of GAD and medication history was also undertaken. Statistical comparison tests such as t-tests, chi-square, and ANOVA were conducted to explore the significance level of the comparison.

\section{Research question and hypothesis}

- Does GAD predispose the severity and probability of neuropathic pain?

- H0: GAD does not significantly predispose the severity and probability of neuropathic pain ( $\mathrm{p}>0.05)$

- H1: GAD significantly predispose the severity and probability of neuropathic pain $(\mathrm{p}<0.05)$.

\section{Results}

The results were expressed as both as descriptive and inferential statistics. The descriptive statistics were used to compute the statistical test of inference. The descriptive statistics that were used in this study is shown in the following Table 1.

However the data depending on the different variables were analyzed for testing to ensure the normal distribution. The data which was non-normal were transformed appropriately as shown in the following table.

\begin{tabular}{|l|l|}
\hline Variables & Mean \pm SD \\
\hline Age & $46 \pm 7.4$ years \\
\hline Vas Score & $5.21 \pm 2.90$ \\
\hline Pain score & $11.90 \pm 5.9$ \\
\hline GAD score & $6.52 \pm 5.3$ \\
\hline
\end{tabular}

Table 1: The descriptive statistics used.

Table 2 is showing the results of Kolmogorov- Smirnov test of the goodness of fit of the normal distribution of this data excluding the VAS scores. Appropriate transformation of the VAS scores was done into the normal distribution through data transformation statistics. These results indicate that validity and reliability of this study is high and the sampling is non-biased. After this, KMO and Bartlett's test were done to check the sampling adequacy, which is displayed in the table below.

\begin{tabular}{|l|l|l|}
\hline Variables & K-S test statistic & P value \\
\hline Age & 0.101 & 0.2 \\
\hline VAS score & 0.169 & 0.001 \\
\hline Pain score & 0.116 & 0.111 \\
\hline GAD score & 0.122 & 0.07 \\
\hline Corrected VAS score & 0.105 & 0.2 \\
\hline
\end{tabular}

Table 2: The results of Kolmogorov- Smirnov test.

The results in Table 3 indicate that the sampling was adequate to explain the model of the study which further confirms the importance of the variables to consider in this model.

\begin{tabular}{|l|l|l|}
\hline \multicolumn{3}{|c|}{ KMO and Bartlett's Test } \\
\hline Kaiser-Meyer-Olkin Measure of Sampling Adequacy & 0.722 \\
\hline \multirow{2}{*}{ Bartlett's Test of Sphericity } & Approx. Chi-Square & 95.597 \\
\cline { 2 - 4 } & Df & 21 \\
\cline { 2 - 4 } & Sig. & 0 \\
\hline
\end{tabular}

Table 3: KMO and Bartlett's test.

Figure 1 states that, categorization of back pain is done by the Pain Detect Questionnaire [12-15], where is it clear that, the nociceptive component of pain is predominant in our study population with respect to the other subgroups.

Figure 2(A) denotes about the actual scenario of the subject population on the basis of medication usage. Majority (43\%) women have reported that, they didn't receive any medications during pain as well as in daily profile. They apply dry heat or ice on the area of pain at the time of pain attack.

Figure 2(B) denotes that, the majority of the subjects are not satisfied with the NSAIDs and they need another means to get relief. 
Citation: Mukhopadhyay A, Chowdhury SSR (2018) Does Generalized Anxiety Disorder (GAD) Predispose the Severity and Probability of the Neuropathic Component of Back Pain? A Cross-sectional Study. J Pain Manage Med 4: 135.

Page 3 of 6

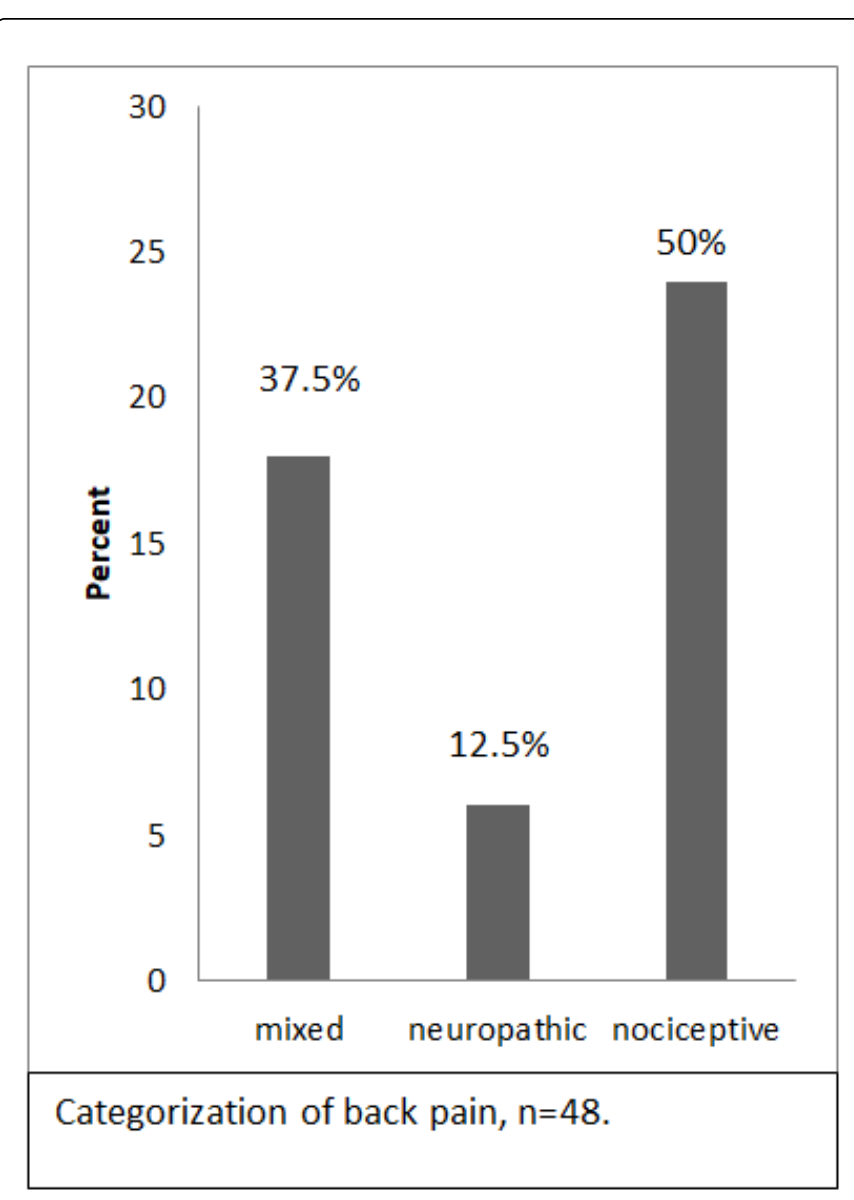

Figure 1: Showing the categorization of reported pain types among the subjects $(\mathrm{n}=48)$.

Figure 2(C) denotes that, majority of the patients having GAD are not properly clinically diagnosed by the physicians and for that, a few subjects are receiving anxiolytic medications along with the pain medication. Though, majority is satisfied with this comedication, but many subjects are not satisfied.

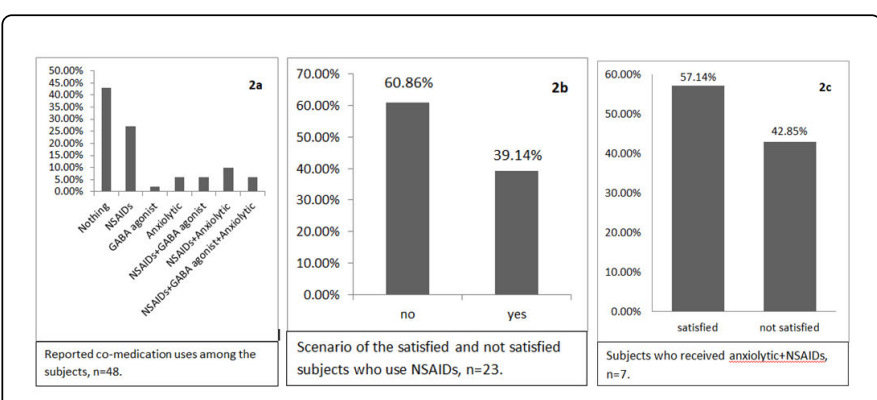

Figure 2: 2a shows the uses of various therapeutic modalities among the subjects. $2 \mathrm{~b}$ Shows the frequency of subjects who are satisfied and not satisfied with the uses of NSAIDs at the time of pain attack $(n=48) .2 \mathrm{c}$ shows satisfaction frequency about their comedication therapy (NSAIDs+Anxiolytics) $(\mathrm{n}=7)$.
Figures 3 and 4 denotes that the low back pain is dominant among the subjects, and also the low back pain is associated sometimes with the upper back as well as with the middle part of the back.

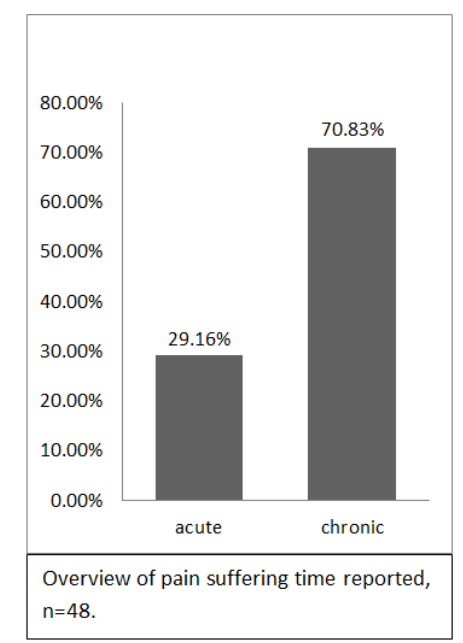

Figure 3: Shows overview of the pain suffering time reported, acute and chronic, $\mathrm{n}=48$.

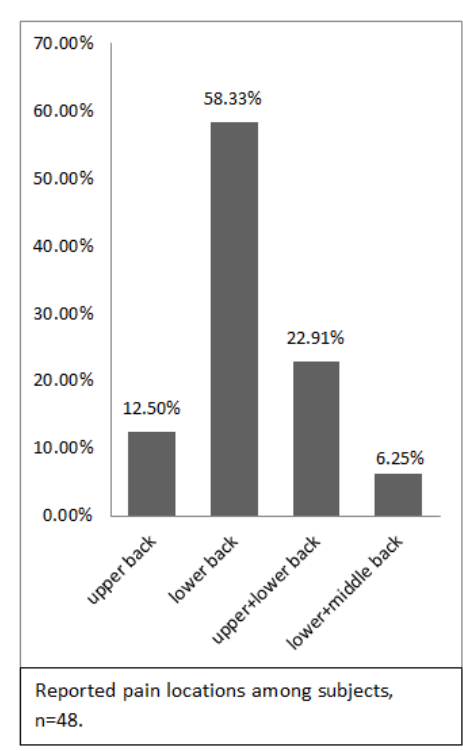

Figure 4: shows pain locations among subjects, $n=48$.

Figure 5 were drawn from the detection procedure of the GAD-7 tool for detecting the Generalized Anxiety Disorder (GAD) as per Spitzer et al. In this case, we only aimed to detect the components of $\mathrm{GAD}$, so other traits of anxiety are not considered here. Categorization is done according to GAD7, a GAD score of $0-4$ seems to be "No GAD", 5-9 seems to be "Mild", and 10-14 seems to be "Moderate" and 15 and above denotes the "Severe" conditions of GA. 
Citation: Mukhopadhyay A, Chowdhury SSR (2018) Does Generalized Anxiety Disorder (GAD) Predispose the Severity and Probability of the Neuropathic Component of Back Pain? A Cross-sectional Study. J Pain Manage Med 4: 135.

Page 4 of 6

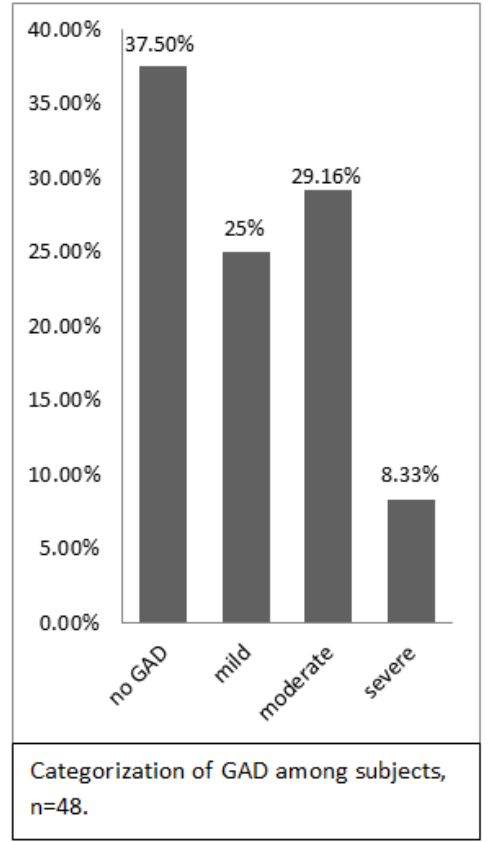

Figure 5: Shows categorization of GAD among the subjects, $n=48$.

Figure 6 denotes the Pain scores and VAS scores are significantly different among the groups (according to the ANOVA results) having no $\mathrm{GAD}$, mild, moderate and severe types of GAD, $\mathrm{P}=0.001$.

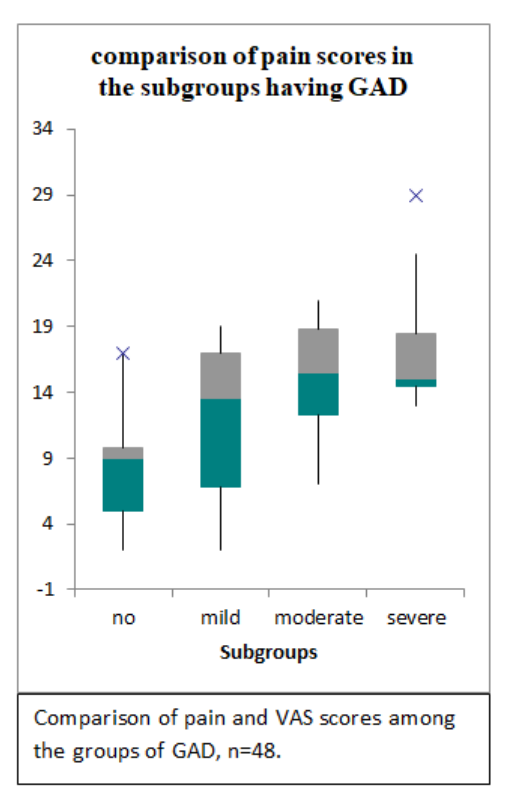

Figure 6: Pain scores and VAS scores are significantly different among the groups (according to the ANOVA results) having no $\mathrm{GAD}$, mild, moderate and severe types of GAD, $\mathrm{P}=0.001$.

Figure 7 denotes significant differences between the groups $\mathrm{P}=0.009$. Results also indicate that, the group using the NSAIDs have higher pain score than the other group, which is found to be significant, $\mathrm{P}=0.004$.

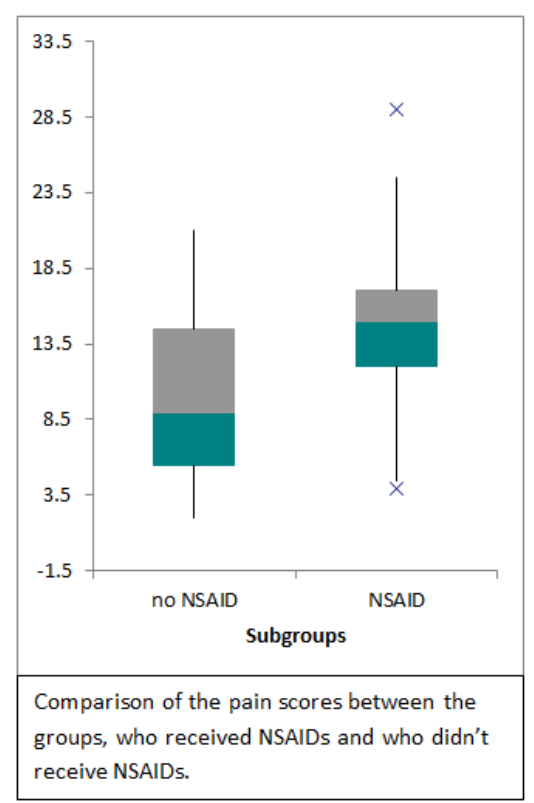

Figure 7: Shows comparison of pain score between the groups who received NSAIDs and who didn't receive NSAIDs.

Table 4 denotes that, age has no significant correlation with the pain score as well as with GAD scores. But the GAD scores are found to be correlated with the pain scores and VAS scores with higher significance level. VAS and pain scores are significantly correlated. Also, it shows that the uses of NSAIDs have a positive correlation with the pain scores which is highly significant.

Regression equation for determining pain scores from GAD scores:

Pain score $=7.837+0.622^{*} \mathrm{GAD}$ score, $(\mathrm{P}=0.000)$.

\begin{tabular}{|l|l|l|}
\hline Variables & Correlation coefficient $(\mathbf{r})$ & P value \\
\hline Pain score and age & -0.054 & 0.71 \\
\hline GAD score and age & 0.126 & 0.39 \\
\hline $\begin{array}{l}\text { GAD score and VAS } \\
\text { score }\end{array}$ & 0.522 & 0 \\
\hline $\begin{array}{l}\text { Pain score and GAD } \\
\text { score }\end{array}$ & 0.559 & 0 \\
\hline $\begin{array}{l}\text { Pain score and VAS } \\
\text { score }\end{array}$ & 0.743 & 0 \\
\hline $\begin{array}{l}\text { Pain scores and uses } \\
\text { of NSAIDs }\end{array}$ & 0.358 & 0.012 \\
\hline
\end{tabular}

Table 4: Shows the correlations of pain scores with various factors.

Table 5 indicates that, GAD significantly increases the risk of neuropathic pain in the subjects than those who didn't report about GAD. Also the risk of neuropathic pain is higher in the NSAID +Anxiolytic managed group. The risk of neuropathic pain is not 
significantly higher in the NSAIDs+GABA agonist+Anxiolytic managed group.

\begin{tabular}{|l|l|}
\hline Variables & Odds risk \\
\hline $\begin{array}{l}\text { Neuropathic pain associated with } \\
\text { GAD }\end{array}$ & 5.2 at $\mathrm{P}=0.0116(1.449-18.714)$ \\
\hline $\begin{array}{l}\text { Risk of neuropathic pain in NSAIDs } \\
\text { managed group }\end{array}$ & 2.04 at $\mathrm{P}=0.3(0.5-8.23)$ \\
\hline $\begin{array}{l}\text { Risk of neuropathic pain in NSAIDs+ } \\
\text { Anxiolytic managed group }\end{array}$ & 12.8 at $\mathrm{P}=0.03(1.14-142.58)$ \\
\hline $\begin{array}{l}\text { Risk of neuropathic pain in NSAIDs }+ \\
\text { Anxiolytic+ GABA agonist managed } \\
\text { group }\end{array}$ & 21 at $\mathrm{P}=0.05(0.93-473.87)$ \\
\hline
\end{tabular}

Table 5: Shows the odds risk of the neuropathic pain among the subjects.

\section{Discussion}

Various types of comorbid diseases and physical conditions can predispose the risk and severity of back pain. Pain is a multi-faceted disease and is influenced by different confounding variables. For example, depression and high BMI (Odds increases the risk of chronic low back pain [9-13]. Correlation between traits of anxiety and pain is both significant and positive [7]. Likewise, cumulative trauma disorders, repetitive strain injuries, non-neutral postures and heavy loads (Awkward postures and loads heavier than RWL leads to pain agony), calcium, vitamin D (low Vitamin D levels and low bone mineral density leads to development of back pain among the menopausal women) and other nutritional deficiencies, hormonal imbalances due to menopause and much more. Different authors reported that a plethora of confounding variables makes it difficult to justify any cause and effect relationship between independent variables that have the probability to increase the severity or perception of back pain.

Hence, the present study was conducted after controlling for the possible confounders of back pain (such as age, height, weight, ethnic group, household status, household income and different forms of surgical and psychopathic interventions). The study showed that GAD was an independent and significant risk factor for predisposing the objective categorization of neuropathy and subjective severity of back pain perception in the study participants. To recall, anxiety is a psychopathic disorder that is featured by serotonergic and adrenergic imbalance within the limbic system of the brain. Excess anxiety will cause sympatho-mimetic discharge during the Fight and Flight phase of the stressors and sensitize both the peripheral and central nerves by upregulating mechanoceptors or proprioceptors. As a result, these receptors would be sensitized to touch, pressure and develop allodynia or hyperalgesia. In a previous study done by the same authors, it was seen that, surgical intervention on the neuronal system can have differential influence on the neuronal sensitization on the Central Nervous System. For example, the Odds Risk of Neuropathic pain among women who received Spinal anesthesia (during C-section delivery) is found to be 1.8 fold higher than the patients who didn't receive Spinal anesthesia $(\mathrm{OR}=1.8 ; \mathrm{P}=0.39)$.

In this study, the majority of patients, i.e. $50 \%$ have reported their nociceptive pain component with respect to mixed pain reporters (37.5\%) and neuropathic pain reporters (12.5\%). Also, back pain seems to be a burden in those patients, because study reports about $70 \%$ cases with chronic pain, from last 5-6 years or more. $47.9 \%$ of the subjects have reported about their usage of various NSAIDs, with or without the prescribed consent from their physicians. In this context, it can be said that the patients who were taking NSAIDs have the significant higher pain scores than who didn't take it. Also, it was found that, majority of the subjects who were receiving the NSAIDs only for the pain relief was not satisfied at all; majority of the subjects belonged to the chronic pain sufferer group. This indicates the need of investigation of other confounding factors along with the pain detection which attributes in pain perceptions. There was a significant correlation between the pain scores and the NSAIDs $(\mathrm{P}=0.012)$ and is addressing that only NSAIDs is insufficient to handle pain among subjects. GAD is found to be an extra burden to the majority of patients (62.5\%). Also a significant correlation of GAD score with pain scores $(\mathrm{P}=0.000)$ and VAS scores $(\mathrm{P}=0.000)$ establish $\mathrm{GAD}$ as a potential component of the back pain perception among the subjects.

The odds of back pain in GAD-affected individuals and in patients on appropriate therapeutic regimes were first elucidated and validated by this present study. According to the results displayed in the Table 5, it can be said that, GAD seems to be a strong risk factor for the subjects for perception of neuropathic pain. Also the results indicated that, the risk is significantly higher in the NSAIDs+ anxiolytic managed group which further indicates the insufficiency of treatment. The risk of neuropathic pain perception is insignificant in the group of NSAIDs+ Anxiolytic + GABA agonist managed group, indicates that the solution of the treatment can be a combined therapy with the said three of the medications in a manner, in which every component of the pain perception would be evaluated and accordingly treated.

Also, it was found that, pain scores and VAS scores varied significantly among the groups having different types of GAD $(\mathrm{P}=0.001)$. All these results support the findings of the studies [4] who stated anxiety as a potential attributer to the pain perception of an individual. We have found that, about $43 \%$ patients who had received the anxioloytic along with NSAIDs were reported as not satisfied with that. This denotes that detection of other comorbid conditions of pain is equally important for the person centric treatment. For this, investigation and treatment through the comprehensive patient history is highly needed. However, studies need to done to find the various correlations among various factors and their roles, those results in pain perception, which leads to affect the pain scores.

\section{References}

1. Castro M, Quarantini L, Daltro C, Caldas M, Koenen K, et al. (2011) Comorbid depression and anxiety symptoms in chronic pain patients and their impact on health related quality of life. Rev Psiq Clin 4: 126-129.

2. Freynhagen R, Baron R, Gockel U, Tölle T (2006) Pain detect: A new screening questionnaire to identify neuropathic components in patients with back pain. Curr Med Res Opin 10: 1911-1920.

3. Gupta G, Nandini N (2015) Prevalence of low back pain in non-working rural housewives of Kanpur, India. Int J Occup Med Environ Health 2: 313-320.

4. Heer E, Gerrits M, Beekman A, Dekker J, Marwijk H, et al. (2014) The association of depression and anxiety with pain: A study from NESDA. PLoS ONE 10: el15077.

5. National Mental Health Survey of India, 2015-16: Prevalence, Pattern and Outcomes by NIMHANS Bangalore, India.

6. Chaudhuri S, Biswas C, Roy K (2012) A subjective and objective analysis of pain in female brick kiln workers of west bengal, India. Int J Occup Med Environ Health 2: 38-43. 
Citation: Mukhopadhyay A, Chowdhury SSR (2018) Does Generalized Anxiety Disorder (GAD) Predispose the Severity and Probability of the Neuropathic Component of Back Pain? A Cross-sectional Study. J Pain Manage Med 4: 135.

Page 6 of 6

7. Santos K, Cendoroglo M, Santos F (2017) Anxiety disorder in elderly persons with chronic pain: frequency and associations. Rev Bras Geriatr Gerontol 21: 91-98.

8. Saya G, Ahdhi G, Subramanian R, Yamuna T (2016) Prevalence of low back pain and its relation to quality of life and disability among women in rural area of Puducherry, India. Indian J Pain 30:111-115.

9. Shiri R, Karppinen A, Arjas P, Solovieva S, Juntura E (2010) The Association between obesity and low back pain: A meta-analysis. Am J Epidemiol 2:135-154.

10. Silva A, Lacativa P, Russo L, Gregório L, Pinheiro R, et al. (2013) Association of back pain with hypovitaminosis D in postmenopausal women with low bone mass. BMC Musculoskelet Disord 14: 184.
11. Spitzer R, Kroenke K, Williams J, Lowe B (2006) A brief measure for assessing generalized anxiety disorder. Arch Inern Med 166: 1092-1097.

12. Tsuji T, Matsudaira K, Sato H, Vietri J (2016) The impact of depression among chronic low back pain patients in Japan. BMC Musculoskelet Disord 17: 447.

13. Vachon-Presseau E, Roy M, Martel M, Caron E, Marin MF, et al. (2013) The stress model of chronic pain: evidence from basal cortisol and hippocampal structure and function in humans. Brain 136: 815-827.

14. Wang Y (2016) Menopause as a potential cause for higher prevalence of low back pain in women than in age matched men. J Orthop Translat 8:1-4.

15. Woo A (2010) Depression and anxiety in pain. Rev Pain 4: 8-12. 